\title{
Salivary Glucose level as Noninvasive Diagnostic Tool for Monitoring Glycemic Control of Type 1 Diabetic Children
}

A.M.Shahin, R.I.Abdel Ati, S.M.Fayd, M.A.G.Mokhtar

Pediatrics and Neonatology Dept., Faculty of Medicine, Benha Univ., Benha, Egypt

E-Mail: Shendy6@gmail.com

\begin{abstract}
Current practices for diabetes management rely on monitoring blood glucose levels. The aim of this study was to estimate the glucose levels of saliva, to assess if there is any significant correlation exists between the serum and salivary glucose levels in children with diabetes mellitus type 1 . This study was a Prospective case control study included 80 Children aged between 4 and 17 years devidied into case group (60 cases) with Confirmed diagnosis of DM type 1 according to the diagnostic criteria by the American Diabetes Association . Controls included 20 healthy controls with matched age and sex compared to cases.All patients were advised to be fasting till both blood and salivary samples were collected.Patients were subjected to the following laboratory investigations:Blood glucose and glycosylated hemoglobin and Salivary glucose. Salivary glucose showed a significant elevation in the saliva in cases compared to controls (3.67 vs. $0.8 \mathrm{mg} / \mathrm{dl}-\mathrm{p}<0.001)$. There was a significant positive correlation between salivary glucose levels and both of serum glucose and HbA1C levels ( $<$ < 0.001). Using a cut off value of $0.93 \mathrm{mg} / \mathrm{dl}$, salivary glucose had sensitivity and specificity of 100 and $85 \%$ respectively in identifying cases with type I diabetes, with an accuracy of $96.25 \%$. salivary glucose is a reliable indicator for both blood glucose and glycosylated hemoglobin levels in patients with type I diabetes mellitus
\end{abstract}

Keywords: Salivary Glucose level, Type I diabetes, Glycosylated hemoglobin.

\section{Introduction}

Egypt is one of the 19 countries and territories of the International Diabetes Federation IDF MENA region. 425 million people have diabetes in the world and more than 39 million people in the MENA Region; by 2045 this will rise to 67 million. There were 8.222.600 cases of diabetes in Egypt in 2017 [1].

Type 1 diabetes mellitus (T1DM), one of the most common chronic diseases in childhood, is caused by insulin deficiency following destruction of the insulinproducing pancreatic beta cells. It most commonly presents in childhood, but one-fourth of cases are diagnosed in adults.so T1DM remains the most common form of diabetes in childhood [2].

Early diagnosis, on-time treatment and continuous management are vital to patients' life quality and to avoid complications. Current practices for diabetes management rely on monitoring blood glucose levels. Blood glucose measurements are required to determine insulin dosage and to detect abnormal glucose levels indicating illnesses, dietary changes, or adverse medication responses. These intrusive tests are generally disliked because of the pain and inconvenience caused by finger pricking, resulting in fewer tests and inadequate blood glucose control. Poor blood glucose control results in more complications and even higher management costs. Particularly, repeated painful finger sticks are a major problem for young children and result in similar negative consequences for disease management but unfortunately Monitoring of blood glucose levels is currently the only recognized and widely used method for the diagnosis and control of diabetes. Thus, a noninvasive, convenient accurate, easyto-use, portable, and low-cost diagnostic tool for diabetes is highly demanded [3].

Human saliva is the secretions by the three major salivary glands(parotid, submandibular ,and sublingual),hundreds of minor salivary glands, and gingival crevice fluid [4]. It consists of water, electrolytes and variety of proteins like enzymes, immunoglobulins, albumin, some polypeptides and biomarkers which can be useful for rapid tests [4].

Saliva is commonly considered as the 'mirror of the body', it is very attractive as a biomedium for clinical diagnostics. Its unique properties, such as noninvasive accessibility and the presence of plentiful disease biomarkers, make it particularly attractive for disease diagnosis and monitoring [5].

Saliva can be easily collected by individuals with modest instruction and it dramatically reduces the discomfort of the tests. Changes in saliva are believed to indicate the wellness of an individual. There are a large number of diagnostic analytes present in saliva, including glucose [5].

The salivary glucose level has been reported to closely reflect blood glucose level prompting it to be used as a diagnostic resource as it offers a distinctive advantage of being a noninvasive procedure allowing multiple sampling [6].

The aim of this study was to estimate the glucose levels of saliva, to assess if there is any significant correlation exists between the serum and salivary glucose levels in children with diabetes mellitus type 1 .

\section{Patient and method}

This study was a Prospective case control study included 80 Children aged between 4 and 17 years devidied into case group (60 cases) with Confirmed diagnosis of DM type 1 according to the diagnostic criteria by the American Diabetes Association (ADA) include the following :A fasting plasma glucose (FPG) level $\geq 126 \mathrm{mg} / \mathrm{dL}(7.0 \mathrm{mmol} / \mathrm{L})$, or A 2-hour plasma 
glucose level $\geq 200 \mathrm{mg} / \mathrm{dL}(11.1 \mathrm{mmol} / \mathrm{L})$ during a $75-\mathrm{g}$ oral glucose tolerance test (OGTT), or a random plasma glucose $\geq 200 \mathrm{mg} / \mathrm{dL}(11.1 \mathrm{mmol} / \mathrm{L})$ in a patient with classic symptoms of hyperglycemia or hyperglycemic crisis. Controls included 20 healthy controls with matched age and sex compared to cases.

Exclusion criteria were Salivary gland disorders, Oral cavity pathologies including oropharyngeal candidiasis, bacterial infections, periodontitis, or viral infections, Antibiotic or corticosteroid therapy for preceding 3 months or any medication other than those for diabetes, Severe diabetic complications, The presence of comorbid diseases and Dental caries.

Every patirnt asked for symptoms of diabetes (Increased thirst, Frequent urination, Bed-wetting in children who previously didn't wet the bed during the night, Extreme hunger, Unintended weight loss, Irritability and other mood changes, Fatigue and weakness and Blurred vision), Dose of insulin, Types and Frequency of insulin administration, Family history of DM and History of any other diseases.

All patients were advised to be fasting till both blood and salivary samples were collected, Patients were subjected to the following laboratory investigations:

- Blood glucose and glycosylated hemoglobin: Venous blood samples were obtained from the subjects for the assessment of blood glucose and hemoglobin A1c levels.

- Salivary glucose: The included subjects were instructed not to brush their teeth 2 hours before sample collection. If the participant was a denture wearer, it was removed before saliva collection. Saliva was collected in a sterile plastic container using the spitting method, when the subject was sitting upright in a comfortable calm room. The collected saliva was immediately transported to clinical pathology laboratory for analysis. The salivary glucose estimation was performed by glucose oxidase-peroxidase (GODPOD) method, End point. 5 $\mu \mathrm{l}$ sample was mixed with $500 \mu \mathrm{l}$ of GOD-POD reagent and incubated at $37 \mathrm{C}$ for $5 \mathrm{~min}$. The glucose was estimated by placing the incubated samples in semi-automated analyzer, and the results were recorded.

We used SPSS software version 25 for Windows 10 for data entry and analysis. At first, data were tested for normality. Quantitative data were expressed as mean and standard deviation, whereas frequency and percentage were used to express categorical variables. For comparison of two independent groups of qualitative data, Chi-Square test (or Fisher's exact test) was performed. Besides, Mann-Whitney $U$ test and independent-Samples t-test and were used to compare two groups of non-parametric and parametric quantitative data respectively.

\section{Results}

The mean age of the included subjects was 11.57 and 10.55 years for cases and controls respectively. Males represented 53.3 and $60 \%$ of subjects in both groups respectively, while the remaining cases were females. Most of the included subjects were from urban areas ( 63.3 and $70 \%$ of subjects in both groups respectively), whereas the remaining subjects were from rural areas. All of the previous demographic data were not significantly different between the two study groups ( $p$ > $0.05)$.

The duration of diabetes ranged between 6 and 36 months in the included cases ( mean $=20.3$ years).

Both serum glucose and glycosylated hemoglobin were significantly elevated in cases compared to controls ( $\mathrm{p}<0.001)$. Serum glucose level had mean values of 288.98 and $87.35 \mathrm{mg} / \mathrm{dl}$ in cases and controls respectively, while $\mathrm{HbA} 1 \mathrm{C}$ had mean values of 8.07 and $4.89 \%$ in the same groups respectively.

Salivary glucose showed a significant elevation in the saliva in cases compared to controls (3.67 vs. $0.8 \mathrm{mg} / \mathrm{dl}-$ $\mathrm{p}<0.001)$.

There was a significant positive correlation between salivary glucose levels and both of serum glucose and HbA1C levels ( $\mathrm{p}<0.001)$.

Using a cut off value of $0.93 \mathrm{mg} / \mathrm{dl}$, salivary glucose had sensitivity and specificity of 100 and $85 \%$ respectively in identifying cases with type I diabetes, with an accuracy of $96.25 \%$.

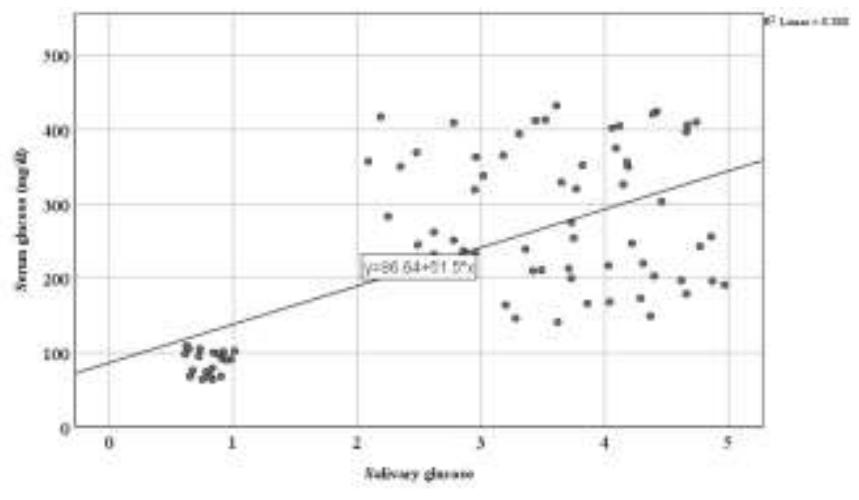

Fig (1) Correlation between salivary and serum glucose levels. 


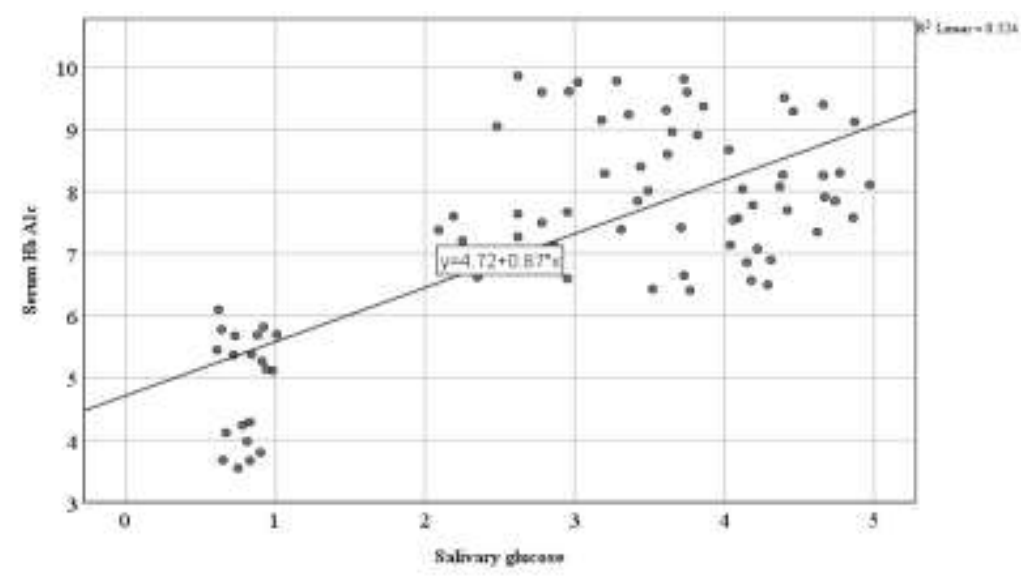

Fig (2) Correlation between Salivary glucose and serum HbA1C levels.

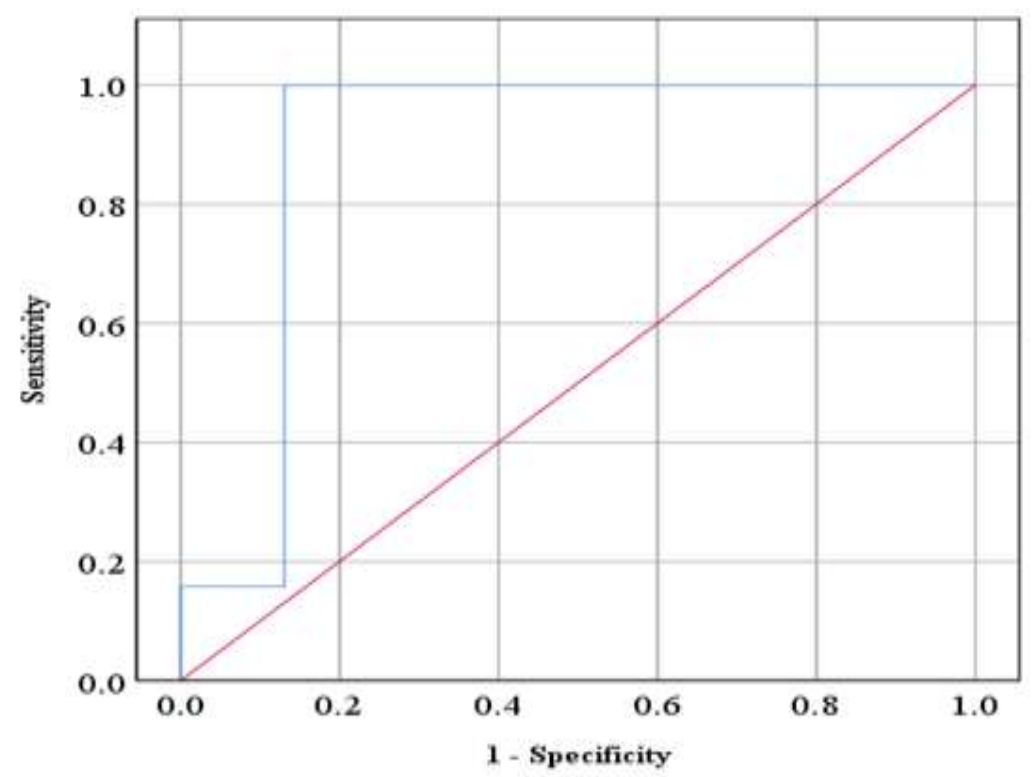

Fig (3) ROC curve for diagnostic profile of salivary levels of Glucose for differentiating diabetic and non-diabetic patients in the current study.

\section{Discussion}

Hemoglobin A1c serves as an indicator of overall glycemic control and a reflection of the average blood sugar over the past three months [7]. As we compared diabetic cases with healthy controls, it was expected that they will have higher HbA1C levels compared to controls, and that agrees with our finding regard glucose levels.

In our study, salivary glucose showed a significant elevation in the saliva in cases compared to controls (3.67 vs. $0.8 \mathrm{mg} / \mathrm{dl}-\mathrm{p}<0.001$ ). Using a cut off value of $0.93 \mathrm{mg} / \mathrm{dl}$, salivary glucose had sensitivity and specificity of 100 and $85 \%$ respectively in identifying cases with type I diabetes, with an accuracy of $96.25 \%$.

In line with our findings, López and his associates reported that salivary glucose levels were significantly elevated in diabetic cases compared to controls (2.05 and $1.03 \mathrm{mg} / \mathrm{dl}$ respectively $-\mathrm{p}<0.05)$ [8]. Shahbaz et al. also reported that salivary glucose had mean values of 2.1 and $0.813 \mathrm{mg} / \mathrm{dl}$ in cases and controls respectively, with highly significant difference between the two groups ( $p<0.001)$ [9]. Moreover, Harisson, Darwazeh, Karjalainen and Lopez reported that the salivary glucose level in diabetic patients was higher than that of healthy subjects $[10,11,12]$.

Behal and Lele reported that the mean level of salivary glucose was reported to be $0.53 \pm 0.4 \mathrm{mg} / \mathrm{dl}$ in controls, $1.14 \pm 1.55 \mathrm{mg} / \mathrm{dl}$ in newly diagnosed diabetics, and $1.22 \mathrm{mg} / \mathrm{dl} \pm 1.99$ in diabetics under treatment. The mean level of salivary glucose in diabetics was significantly higher than that in non-diabetics [13].

Panchbhai and his associates reported that salivary glucose levels had higher values in uncontrolled diabetic cases $(8.09 \mathrm{mg} / \mathrm{dl})$, compared to controlled diabetic cases (7.64 mg/dl), and controls (1.89 mg/dl) [14].Other authors reported the same findings [15]. Additionally, Sharon and his associates reported that salivary glucose was significantly elevated only in the parotid saliva [15].

Saliva contains serumnal components transported from blood capillaries into saliva by diffusion, active transport, and/or ultra- filtration via gingival sulcus. Hence, saliva can serve as a partial filtrate of blood for 
monitoring the health status of a person [16].

The rationale for the use of saliva in monitoring hyperglycaemia could have many explanations. Glucose is a small molecule which can easily diffuse through semipermeable membranes. The alteration in basement membrane of blood vessels (in patients with diabetes mellitus) leads to increased diffusion of glucose from blood to saliva [9]. Thus, large amounts of glucose concentrate in saliva of patients with diabetes mellitus [17].

Moreover, salivary glands act as filters of blood glucose that would be altered by hormonal or neural regulations [18]. under several conditions of stress in humans $[12,18]$. Hence, salivary glucose level decreased after beginning insulin treatment in both children and adolescents [12], while infections (e.g candidiasis in those with dentures or HIV) and inflammation of salivary glands could increase the glucose level in saliva [19].

On the contrary, Vaziri and his associates negated any significant difference between cases with type I diabetes and controls as regard salivary glucose levels ( $p$ $=0.88$ ). It had mean levels of 14.52 and $14.82 \mathrm{mg} / \mathrm{dl}$ in both groups respectively [20]. Furthermore, Marchetti et al. reported no changes in the salivary glucose levels in diabetic personnel [21].

In the current study, there was a significant positive correlation between salivary glucose levels and blood serum glucose levels $(r=0.623-\mathrm{p}<0.001)$.

Panchbhai et al. confirmed our findings as there was a significant positive correlation between salivary and serum glucose levels $(p=0.04)$ [14]. Amer also demonstrated a significant relationship between salivary and blood glucose levels and recommended using saliva for determination of blood glucose level in diabetic patients [22].

Additionally, another study reported that there was a significant correlation observed between salivary and serum glucose levels. The Karl Pierson co-efficient for correlation between serum and salivary glucose in T1DM group was $r=0.984$ with a $p$ value $<0.001$ [9].

In another study, a significant correlation was seen between serum and salivary glucose levels in type $1 \mathrm{DM}$ and also in healthy controls using Pearson's correlation test [19]. Another study handling uncontrolled diabetics, the salivary glucose levels show a nearly perfect correlation coefficient $(\mathrm{r}=0.929)$ and a statistically strong significant association $(\mathrm{P}<0.001)$ with blood glucose levels [23].

However, Leach and Ficara found no significant correlation between blood glucose and salivary glucose in diabetic patients [24]. This difference may be due to the different methodology and design of their study compared to ours and the fact that it was not clear what food products had been consumed by subjects within the two hours prior to sampling. Moreover, this difference may be attributed to the release of carbohydrates by glycoproteins, glucose break down by the bacteria and salivary contamination by crevicular fluid exudate in patients with poor oral hygiene [25].
In the current study, there was a significant positive correlation between salivary glucose levels and HbA1C levels $(r=0.724-p<0.001)$.

In another study, A nearly perfect correlation coefficient $(r=0.906)$ and a statistically strong significant association $(\mathrm{P}<0.001)$ was seen between salivary glucose levels and glycosylated hemoglobin [23]. This agrees with our findings.

Moreover, a study conducted by Panda et al. showed a high correlation between salivary glucose levels and glycosylated hemoglobin in controlled diabetics, which is also similar to our study [26].

In contrast to our study, Lopez et al. did not find any correlation between salivary glucose level and HbAlc percentage [8].

Our study has some limitations; first of all; it is a single center study that included a relatively small sample size. Therefore. More studies including more cases from different pediatric endocrinological centers should be conducted in the near future.

\section{Conclusion}

Based on our results, it appears that salivary glucose is a reliable indicator for both blood glucose and glycosylated hemoglobin levels in patients with type I diabetes mellitus. It represents a noninvasive tool for diagnosis and monitoring of such cases.

\section{References}

[1] B.Viswanath,; C. S.Choi,; K.Lee, S. Kim. Recent trends in the development of diagnostic tools for diabetes mellitus using patient saliva. TrAC Trends in Analytical Chemistry. Vol. 89,pp. 60-67, 2017.

[2] C.Tóth, Z.Clemens. A child with type 1 diabetes mellitus (T1DM) successfully treated with the Paleolithic ketogenic diet: A 19-month insulin freedom. Int J Case Rep Images. Vol. 6(12),pp. 752-757, 2015.

[3] W.Zhang, Y.Du, M. L.Wang. Noninvasive glucose monitoring using saliva nano-biosensor. Sensing and Bio-Sensing Research. Vol. 4,pp. 23-29, 2015.

[4] J.Gilloteaux, A.Afolayan. Clarification of the terminology of the major human salivary glands: acinus and alveolus are not synonymous. The Anatomical Record. Vol. 297(8),pp. 1354-1363, 2014.

[5] C.Nöhammer. Saliva a sample matrix for systemic disease diagnostics. Clinical Oral Implants Research. Vol. 29,pp. 39-39, 2018.

[6] A.Gupta, S. K.Singh, B.Padmavathi, S.Rajan, G.Mamatha, S.Kumar, et al. Evaluation of correlation of blood glucose and salivary glucose level in known diabetic patients. Journal of Clinical and Diagnostic Research: JCDR. Vol. 9(5), pp.106, 2015.

[7] S. I.Sherwani, H. A.Khan, A.Ekhzaimy, A.Masood, M. K.Sakharkar, Significance of HbAlc test in diagnosis and prognosis of diabetic patients. Biomarker insights, 11, BMI. S38440, 2016.

[8] M. E.López, M. E.Colloca, R. G.Páez, J. N. 
Schallmach, M. A.Koss, A. Chervonagura. Salivary characteristics of diabetic children. Brazilian dental journal. Vol. 14(1),pp. 26-31, 2003.

[9] S.Shahbaz, G.Katti, S. R. Ghali, C.Katti, D. D.Diwakar, V. Guduba. Salivary alterations in type 1 diabetes mellitus patients: salivary glucose could be noninvasive tool for monitoring diabetes mellitus. Indian Journal of Dental Research. Vol. 25(4),pp. 420, (2014).

[10] A.Darwazeh, T.MacFarlane, A.McCuish, P. J.Lamey. Mixed salivary glucose levels and candidal carriage in patients with diabetes mellitus. Journal of oral pathology \& medicine. Vol. 20(6),pp. 280-283, 1991.

[11] R.Harrison, W. H.Bowen. Flow rate and organic constituents of whole saliva in insulin-dependent diabetic children and adolescents. Pediatr Dent. Vol. 9(4),pp. 287-291, 1987.

[12] K. M.Karjalainen, M. Knuuttila, M. Käär. Salivary factors in children and adolescents with insulindependent diabetes mellitus. Pediatric dentistry. Vol. 18(4),pp. 306-311, 1996.

[13] S.Behal, S.Lele. Correlation of Salivary Glucose with Blood Glucose in Diabetic Individuals. Indian Journal of Stomatology. Vol. 3(4), 2012.

[14]A. S.Panchbhai. S. S.Degwekar, R. R. Bhowte. Estimation of salivary glucose, salivary amylase, salivary total protein and salivary flow rate in diabetics in India. Journal of oral science. Vol. 52(3),pp. 359-368, 2010.

[15] A.Sharon, H.Ben-Aryeh, B.Itzhak, K.Yoram, R.Szargel, D.Gutman. Salivary composition in diabetic patients. Journal of oral medicine. Vol. 40(1),pp. 23, 1985.

[16]P.Khanna, D. R.Walt. Salivary diagnostics using a portable point-of-service platform: a review. Clinical therapeutics. Vol. 37(3),pp. 498-504, 2015.

[17] C.Naing, J. W.Mak. Salivary glucose in monitoring glycaemia in patients with type 1 diabetes mellitus: a systematic review. Journal of Diabetes \& Metabolic Disorders. Vol. 16(1),pp. 2, 2017.

[18] R. T.Chatterton Jr. K. M.Vogelsong, Y. c.Lu, A. B.Ellman, G. A.Hudgens. Salivary $\alpha$-amylase as a measure of endogenous adrenergic activity. Clinical physiology. Vol. 16(4),pp. 433-448, 1996.

[19] V.Nagalaxmi, V.Priyanka. Can saliva be a marker for predicting type 1 diabetes mellitus?-A pilot study. Journal of Indian Academy of Oral Medicine and Radiology. Vol. 23(4),pp. 579, 2011.

[20]P. B.Vaziri, M.Vahedi, H.Mortazavi, S.Abdollahzadeh, M. Hajilooi. Evaluation of salivary glucose, IgA and flow rate in diabetic patients: a case-control study. Journal of Dentistry (Tehran, Iran). Vol. 7(1),pp. 13, 2010.

[21] P.Marchetti, M.Tognarelli, R.Giannarelli, C.Grossi, L.Picaro, A.di Carlo, et al. Decreased salivary glucose secretory rate: usefulness for detection of diabetic patients with autonomic neuropathy. Diabetes research and clinical practice. Vol. 7(3),pp. 181-186, 1989.

[22] S.Amer, M.Yousuf, P.Siddqiui, J.Alam. Salivary glucose concentrations in patients with diabetes mellitus-a minimally invasive technique for monitoring blood glucose levels. Pak J Pharm Sci. vol. 14(1), pp. 33-37, 2001.

[23] S.Kumar, S.Padmashree, R.Jayalekshmi. Correlation of salivary glucose, blood glucose and oral candidal carriage in the saliva of type 2 diabetics: A case-control study. Contemporary clinical dentistry. Vol. 5(3), pp.312, 2014.

[24] A. J.Ficara, M. P.Levin, M.Grower, G. D.Kramer. A comparison of the glucose and protein content of gingival fluid from diabetics and nondiabetics. Journal of periodontal research. Vol. 10(3),pp. 171 $175,1975$.

[25] C.Jurysta, N.Bulur, B.Oguzhan, I.Satman, T. M.Yilmaz, W. J.Malaisse, et al. Salivary glucose concentration and excretion in normal and diabetic subjects. Journal of Biomedicine and Biotechnology, 2009.

[26]P.Abikshyeet, V.Ramesh, N.Oza. Glucose estimation in the salivary secretion of diabetes mellitus patients. Diabetes, metabolic syndrome and obesity: targets and therapy. vol. 5 ,pp. 149, 2012. 